\title{
Torque Limit of Horizontal Axis Wind Turbine
}

\author{
Haibo Jiang \\ Logistics College \\ Naval University of Engineering \\ Tianjin 300450, China \\ jianghaibo022@126.com
}

\author{
Zhongqing Cheng \\ Logistics College \\ Naval University of Engineering \\ Tianjin 300450, China \\ bace@tom.com
}

\author{
Yunpeng Zhao \\ Logistics College \\ Naval University of Engineering \\ Tianjin 300450, China \\ sohubaiyan@sohu.com
}

\begin{abstract}
The torque coefficient of any wind turbine must have highest limit. In this paper, an analytical expression of torque coefficient associated with tip speed ratio and airfoil lift drag ratio of wind turbine with ideal chord has been deduced by integrating along the blade wingspan using the blade element momentum theory, which can be used for pre-estimating torque coefficient of actual wind turbine in design. Further, considering ideal fluid environment ( the drag coefficient is close to 0 ), an expression of the highest performance of torque only associated with tip speed ratio has been deduced too, which is the highest boundary of torque coefficient of any actual wind turbine with same tip speed ratio. The results show that for the wind turbine in steady operation state when the tip speed ratio is about $\mathbf{0 . 6 3 5}$, there is a theoretical limit of the torque coefficient, 0.401 ; if the tip speed ratio is greater than 6 , the torque coefficient is unlikely to exceed 0.1 .
\end{abstract}

Keywords-wind energy utilization; horizontal axis wind turbine; torque coefficient; torque limit; highest performance; lift drag ratio; tip speed ratio

\section{INTRODUCTION}

The most important performances of horizontal axis wind turbine are power, torque and thrust. Power coefficient has been reported in extensive literatures. The theoretical limit of the power coefficient deduced by Betz is $16 / 27$, or about $0.593^{[1-2]}$. Reference [3] deduced the formula of the highest power coefficient related with the lift drag ratio and the tip speed ratio of the wind turbine in the steady operation state, and proved that power coefficient will be close to the Betz limit only when the tip speed ratio and the lift drag ratio are close to infinite, So people do not have to spend too much energy to pursue the power coefficient beyond the theoretical limit. In course of design, if wind turbine efficiency is close to the theoretical value under the corresponding lift drag ratio and tip speed ratio, the design can be identified as the "ideal design". The theoretical value is the goal of actual design.

Corresponding to the wind turbine power coefficient, another important property of the wind turbine is torque coefficient. Is there a theoretical limit about the torque coefficient? This paper will give a positive answer. This paper will deduce the formula of the highest torque coefficient related with the lift drag ratio and the tip speed ratio of the wind turbine in the steady operation state by the blade element - momentum theory.

\section{THE BASIC RELATIONSHIP FORMULA IN STEADY OPERATION STATE}

First we investigate the action of the wind and the blade force state in wind turbine steady operation state, or neither accelerated nor decelerated running state (see Figure 1 and Figure 2). If the blade was cut with concentric cylinder, the cutting surface position is A-A section shown in Figure 1. A blade element $\mathrm{d} r$ taken at a radius $r$ (the A-A position) is shown in Figure 2.

The meaning of the symbols in the figures is defined as follows.

$\omega$ is the rotation angular velocity of the wind turbine ( blade element dr upward along the rotation plane ). $W$ is the relative velocity, $W=\omega r . b$ is the tangential velocity inducing factor. $a$ is the axial velocity inducing factor. $U$ is the absolute speed of wind. $u$ is the axial velocity through the blade, $u=$ (1-a) $U . w$ is the synthetic speed of the relative velocity $W$ and the tangential speed of induction $b W, w=(1+b) W . v$ is the synthetic speed of $u$ and $w . \alpha$ is the attack angle ofvelocity $v . \mathrm{d} L$ is the lift of the blade element. $\mathrm{d} L_{w}$ is the circumferential component of lift $\mathrm{d} L . \mathrm{d} L_{u}$ is the horizontalcomponent of the lift $\mathrm{d} L . \mathrm{d} D$ is the drag of the blade element, consistent with the direction of $v . \mathrm{d} D_{w}$ is the circumferential component of the drag $\mathrm{d} D . \mathrm{d} D_{u}$ is the horizontal component of the drag $\mathrm{d} D$.

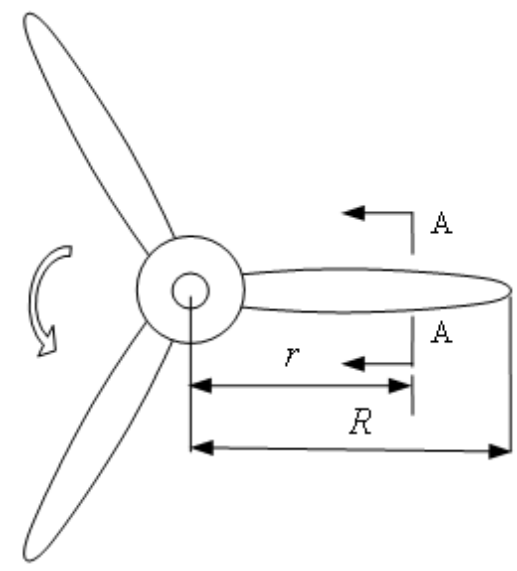

Figure 1. The schematic diagram of wind turbine impeller in downwind direction 


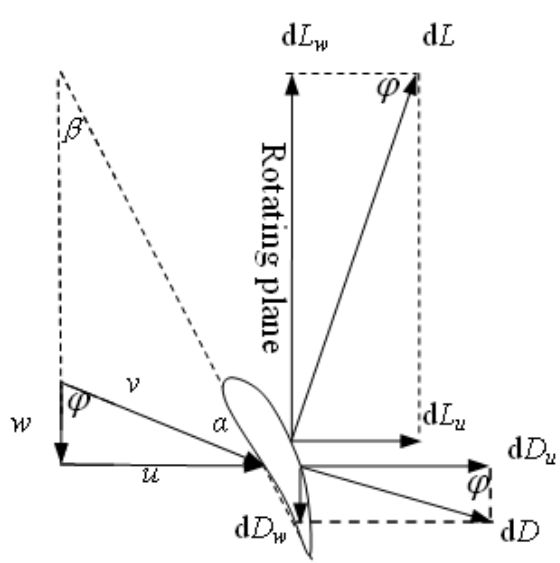

Figure 2. Wind speed state and force analysis of the blade element at AAsection in Figure 1

According to Figure 2, the inflow angle $\varphi$ is determined by the following formula

$$
\tan \varphi=\frac{u}{w}=\frac{(1-a) U}{(1+b) \omega r}=\frac{1-a}{1+b} \frac{1}{\lambda}
$$

In steady operation state, the axial velocity inducing factor $a$ and the circumferential speed of inducing factor $b$ exist stable value ${ }^{[4]}$ :

$$
a=\frac{1}{3}, \quad b=\frac{a(1-a)}{\lambda^{2}}=\frac{2}{9 \lambda^{2}}
$$

So

$$
\begin{aligned}
& \tan \varphi=\frac{1-a}{1+b} \frac{1}{\lambda}=\frac{1-a}{1+\frac{a(1-a)}{\lambda^{2}}} \frac{1}{\lambda}=\frac{6 \lambda}{9 \lambda^{2}+2} \\
& =\frac{6 \lambda_{t}(r / R)}{9 \lambda_{t}^{2}(r / R)^{2}+2}
\end{aligned}
$$

Where $R$ is the length of the blade; $\lambda$ is the ratio of the tangential line speed $W$ at $r$ and the absolute wind speed $U$, known as the linear speed ratio; $\lambda_{t}$ is the tip speed ratio, the conversion formula is $\lambda=\lambda_{t} r / R$. $\lambda_{t}$ was preset in design.

The inflow speed can be obtained from Figure 2

$$
\begin{aligned}
& v=\sqrt{w^{2}+u^{2}}=\sqrt{(1+b)^{2} W^{2}+(1-a)^{2} U^{2}} \\
& =U \sqrt{\left(1+\frac{2}{9 \lambda^{2}}\right)^{2} \lambda^{2}+\left(1-\frac{1}{3}\right)^{2}} \\
& =U \sqrt{\left(\lambda+\frac{2}{9 \lambda}\right)^{2}+\left(\frac{2}{3}\right)^{2}}
\end{aligned}
$$

The sine and cosine expressions of the inflow angle are

$$
\begin{aligned}
& \sin \varphi=\frac{u}{v}=\frac{\left(1-\frac{1}{3}\right) U}{U \sqrt{\left(\lambda+\frac{2}{9 \lambda}\right)^{2}+\left(\frac{2}{3}\right)^{2}}}=\frac{\frac{2}{3}}{\sqrt{\left(\lambda+\frac{2}{9 \lambda}\right)^{2}+\left(\frac{2}{3}\right)^{2}}} \\
& \cos \varphi=\frac{w}{v}=\frac{\left(1+\frac{2}{9 \lambda^{2}}\right) \omega r}{U \sqrt{\left(\lambda+\frac{2}{9 \lambda}\right)^{2}+\left(\frac{2}{3}\right)^{2}}}=\frac{\lambda+\frac{2}{9 \lambda}}{\sqrt{\left(\lambda+\frac{2}{9 \lambda}\right)^{2}+\left(\frac{2}{3}\right)^{2}}}
\end{aligned}
$$

The aerodynamic performance analytical formulas of wind turbine in steady operation state can be deduced from these basic relationships.

\section{TORQUE COEFFICIENT FORMULA DERIVATION}

Let $\rho$ is air density, $C$ is the chord length of the blade element, $C_{L}$ is airfoil lift coefficient, $C_{D}$ is the airfoil drag coefficient. The blade element torque formula could be deduced based on the basis of the aforementioned formulas and blade element theory ${ }^{[5-6]}$.

According to lift definition, the blade element lift expression is

$$
\begin{aligned}
& \mathrm{d} L=\frac{1}{2} \rho v^{2} C \cdot C_{L} \mathrm{~d} r=\frac{1}{2} \rho\left[U \sqrt{\left(\lambda+\frac{2}{9 \lambda}\right)^{2}+\left(\frac{2}{3}\right)^{2}}\right]^{2} C \cdot C_{L} \mathrm{~d} r \\
& =\frac{1}{2} \rho U^{2} C C_{L}\left[\left(\lambda+\frac{2}{9 \lambda}\right)^{2}+\left(\frac{2}{3}\right)^{2}\right] \mathrm{d} r
\end{aligned}
$$

Similarly blade element drag expression is

$$
\begin{aligned}
& \mathrm{d} D=\frac{1}{2} \rho v^{2} C \cdot C_{D} d r=\frac{1}{2} \rho\left[U \sqrt{\left(\lambda+\frac{2}{9 \lambda}\right)^{2}+\left(\frac{2}{3}\right)^{2}}\right]^{2} C \cdot C_{D} \mathrm{~d} r \\
& =\frac{1}{2} \rho U^{2} C C_{D}\left[\left(\lambda+\frac{2}{9 \lambda}\right)^{2}+\left(\frac{2}{3}\right)^{2}\right] \mathrm{d} r
\end{aligned}
$$

According to Figure 2, total tangential lift $\mathrm{d} F$ can be obtained by the formula (5-8)

$$
\begin{aligned}
& \mathrm{d} F=\mathrm{d} L_{w}-\mathrm{d} D_{w}=\mathrm{d} L \sin \varphi-\mathrm{d} D \cos \varphi \\
& =\frac{1}{2} \rho U^{2} C C_{L}\left[\left(\lambda+\frac{2}{9 \lambda}\right)^{2}+\left(\frac{2}{3}\right)^{2}\right] \frac{\frac{2}{3}}{\sqrt{\left(\lambda+\frac{2}{9 \lambda}\right)^{2}+\left(\frac{2}{3}\right)^{2}}} \mathrm{~d} r- \\
& \frac{1}{2} \rho U^{2} C C_{D}\left[\left(\lambda+\frac{2}{9 \lambda}\right)^{2}+\left(\frac{2}{3}\right)^{2}\right] \frac{\lambda+\frac{2}{9 \lambda}}{\sqrt{\left(\lambda+\frac{2}{9 \lambda}\right)^{2}+\left(\frac{2}{3}\right)^{2}}} \mathrm{~d} r \\
& =\frac{1}{2} \rho U^{2} C\left[\frac{2}{3} C_{L}-\left(\lambda+\frac{2}{9 \lambda}\right) C_{D}\right] \sqrt{\left(\lambda+\frac{2}{9 \lambda}\right)^{2}+\left(\frac{2}{3}\right)^{2}} \mathrm{~d} r
\end{aligned}
$$

The total torque of the blade element can be obtained by formula (9) 


$$
\mathrm{d} M=r \mathrm{~d} F=\frac{1}{2} \rho U^{2} C\left[\frac{2}{3} C_{L}-\left(\lambda+\frac{2}{9 \lambda}\right) C_{D}\right] \sqrt{\left(\lambda+\frac{2}{9 \lambda}\right)^{2}+\left(\frac{2}{3}\right)^{2}} r \mathrm{~d} r
$$

The wind turbine is composed by several blades; the total torque formula can be obtained by integration. Let $B$ is the number of blades of the wind turbine, and $x$ represents the relative chord length $r / R$. From formula (10), the integral formula of wind turbine torque coefficient is

$$
\begin{aligned}
& C_{M}=\frac{B}{\frac{1}{2} \rho U^{2} \pi R^{3}} \int_{R} \frac{1}{2} \rho U^{2} C\left[\frac{2}{3} C_{L}-\left(\lambda+\frac{2}{9 \lambda}\right) C_{D}\right] \sqrt{\left(\lambda+\frac{2}{9 \lambda}\right)^{2}+\left(\frac{2}{3}\right)^{2}} r \mathrm{~d} r \\
& =\frac{B}{\pi} \int_{0}^{1}\left(\frac{r}{R}\right)\left(\frac{C}{R}\right)\left[\frac{2}{3} C_{L}-\left(\lambda+\frac{2}{9 \lambda}\right) C_{D}\right] \sqrt{\left(\lambda+\frac{2}{9 \lambda}\right)^{2}+\left(\frac{2}{3}\right)^{2}} \mathrm{~d}\left(\frac{r}{R}\right) \\
& =\frac{B}{\pi} \int_{0}^{1} x\left(\frac{C}{R}\right)\left[\frac{2}{3} C_{L}-\left(\lambda_{t} x+\frac{2}{9 \lambda_{t} x}\right) C_{D}\right] \sqrt{\left(\lambda_{t} x+\frac{2}{9 \lambda_{t} x}\right)^{2}+\left(\frac{2}{3}\right)^{2}} \mathrm{~d} x
\end{aligned}
$$

References $[3,4,5]$ have given the expression of the ideal chord $C$

$$
\frac{C}{R}=\frac{16 \pi}{9 B} \frac{x}{\left[\left(\lambda_{t} x+\frac{2}{9 \lambda_{t} x}\right) C_{L}+\frac{2}{3} C_{D}\right] \sqrt{\left(\lambda_{t} x+\frac{2}{9 \lambda_{t} x}\right)^{2}+\left(\frac{2}{3}\right)^{2}}}
$$

The ideal chord length derived form blade element momentum theory. By the definition of the ratio $\zeta$ of airfoil lift to drag, $\zeta=C_{L} / C_{D}$.

The wind turbine torque coefficient integral formula can be obtained by the formula (11) and (12)

$$
\begin{aligned}
& C_{M}=\frac{B}{\pi} \int_{0}^{1} x\left(\frac{C}{R}\right)\left[\frac{2}{3} C_{L}-\left(\lambda_{t} x+\frac{2}{9 \lambda_{t} x}\right) C_{D}\right] \sqrt{\left(\lambda_{t} x+\frac{2}{9 \lambda_{t} x}\right)^{2}+\left(\frac{2}{3}\right)^{2}} \mathrm{~d} x \\
& =\frac{B}{\pi} \int_{0}^{1} x\left\{\frac{16 \pi}{9 B} \frac{x}{\left[\left(\lambda_{t} x+\frac{2}{9 \lambda_{t} x}\right) C_{L}+\frac{2}{3} C_{D}\right] \sqrt{\left(\lambda_{t} x+\frac{2}{9 \lambda_{t} x}\right)^{2}+\left(\frac{2}{3}\right)^{2}}}\right\} \\
& =\frac{16}{9} \int_{0}^{1} \frac{x^{2}\left[\frac{2}{3} C_{L}-\left(\lambda_{t} x+\frac{2}{9 \lambda_{t} x}\right) C_{D}\right] \sqrt{\left(\lambda_{t} x+\frac{2}{9 \lambda_{t} x}\right)^{2}+\left(\frac{2}{3}\right)^{2}} \mathrm{~d} x}{\left(\lambda_{t} x+\frac{2}{9 \lambda_{t} x}\right) C_{L}+\frac{2}{3} C_{D}} \mathrm{~d} x \\
& =\frac{16}{9} \int_{0}^{1} \frac{x^{2}\left[\frac{2}{3} \zeta-\left(\lambda_{t} x+\frac{2}{9 \lambda_{t} x}\right)\right]}{\left(\lambda_{t} x+\frac{2}{9 \lambda_{t} x}\right) \zeta+\frac{2}{3}} \mathrm{~d} x
\end{aligned}
$$

\section{THE GENERAL PERFORMANCE OF THE WIND TURBINE TORQUE}

The general performance of the torque is torque coefficient associated with lift drag ratio and tip speed ratio. The coefficient can be obtained by integration of formula (13)

$$
\begin{aligned}
C_{M}= & \frac{16}{9} \int_{0}^{1} \frac{x^{2}\left[\frac{2}{3} \zeta-\left(\lambda_{t} x+\frac{2}{9 \lambda_{t} x}\right)\right]}{\left(\lambda_{t} x+\frac{2}{9 \lambda_{t} x}\right) \zeta+\frac{2}{3}} \mathrm{~d} x \\
= & \frac{16\left(-4-4 \zeta^{2}+3 \zeta \lambda_{t}+3 \zeta^{3} \lambda_{t}-3 \zeta^{2} \lambda_{t}^{2}\right)}{81 \zeta^{3} \lambda_{t}^{2}}- \\
& \frac{32\left(-2-\zeta^{2}+\zeta^{4}\right)}{243 \zeta^{4} \lambda_{t}^{3}}\left[\ln (2 \zeta)-\ln \left(2 \zeta+6 \lambda+9 \zeta \lambda_{t}^{2}\right)\right]+ \\
& \frac{64\left(-2+\zeta^{2}+3 \zeta^{4}\right)}{243 \zeta^{4} \lambda_{t}^{3} \sqrt{-1+2 \zeta^{2}}}\left(\operatorname{arccot} \sqrt{-1+2 \zeta^{2}}-\arctan \frac{1+3 \zeta \lambda_{t}}{\sqrt{-1+2 \zeta^{2}}}\right)
\end{aligned}
$$

The torque coefficient $C_{M}$ changes only with the tip speed ratio $\lambda_{t}$ and lift drag ratio $\zeta$. Some data have been showed in Table 1. These data can be used as the reference value of the actual wind turbine torque coefficient.

TABLE I. THE THEORETICAL VALUE OF THE TORQUE COEFFICIENT OF THE WIND TURBINE WITH IDEAL CHORD LENGTH

\begin{tabular}{|c|c|c|c|c|c|}
\hline \multirow{2}{*}{$\begin{array}{c}\text { tip speed } \\
\text { ratio, } \lambda_{\boldsymbol{t}}\end{array}$} & \multicolumn{5}{|c|}{ Lift drag Ratio, $\zeta$} \\
\cline { 2 - 6 } & $\mathbf{1 0}$ & $\mathbf{5 0}$ & $\mathbf{1 0 0}$ & $\mathbf{5 0 0}$ & $\mathbf{1 0 0 0}$ \\
\hline 1 & 0.291 & 0.352 & 0.36 & 0.366 & 0.367 \\
\hline 2 & 0.181 & 0.234 & 0.241 & 0.246 & 0.247 \\
\hline 3 & 0.116 & 0.166 & 0.173 & 0.178 & 0.179 \\
\hline 4 & 0.078 & 0.127 & 0.133 & 0.138 & 0.139 \\
\hline 5 & 0.053 & 0.101 & 0.107 & 0.112 & 0.113 \\
\hline 6 & 0.036 & 0.083 & 0.09 & 0.094 & 0.095 \\
\hline 7 & 0.023 & 0.07 & 0.077 & 0.081 & 0.082 \\
\hline 8 & 0.013 & 0.061 & 0.067 & 0.071 & 0.072 \\
\hline 9 & 0.005 & 0.053 & 0.059 & 0.064 & 0.064 \\
\hline 10 & $/$ & 0.046 & 0.052 & 0.057 & 0.058 \\
\hline
\end{tabular}

\section{THE HIGHEST PERFORMANCE OF TORQUE}

The highest performance of the torque appears only when the drag coefficient $C_{D}$ is equal to 0 in ideal fluid environment, which is only the function of tip speed ratio. In formula (13), Order $C_{D}=0$ or $\zeta \rightarrow \infty$, the formula (13) can be integrated,

$$
\begin{aligned}
& C_{M \max }=\frac{16}{9} \int_{0}^{1} \frac{\frac{2}{3} x^{2}}{\left(\lambda_{t} x+\frac{2}{9 \lambda_{t} x}\right)} \mathrm{d} x \\
& =\frac{16}{243 \lambda_{t}^{3}}\left[9 \lambda_{t}^{2}-2 \ln \left(9 \lambda_{t}^{2}+2\right)+2 \ln 2\right]
\end{aligned}
$$

The curve of the highest performance of the torque corresponding to the tip speed ratio was shown in Figure 3, and the calculated values as shown in Table 2. 


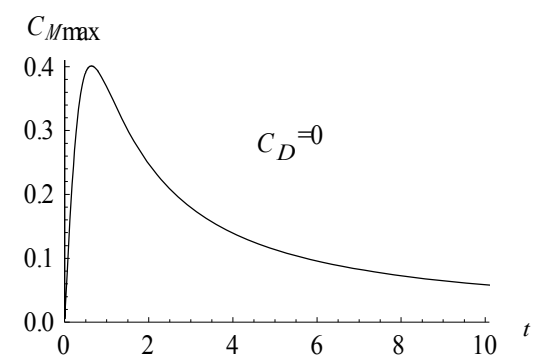

Figure 3. The highest performance curve of the torque coefficient only associated with the tip speed ratio

TABLE II. THE THEORETICAL VALUES OF THE TORQUE COEFFICIENT ONLY ASSOCIATED WITH THE TIP SPEED RATIO

\begin{tabular}{|c|c|}
\hline tip speed ratio, $\boldsymbol{\lambda}_{\boldsymbol{t}}$ & torque highest performance, $\boldsymbol{C}_{\text {Mmax }}$ \\
\hline 1.0 & 0.368099 \\
\hline 2.0 & 0.247828 \\
\hline 3.0 & 0.179360 \\
\hline 4.0 & 0.139320 \\
\hline 5.0 & 0.113534 \\
\hline 6.0 & 0.095660 \\
\hline 7.0 & 0.082583 \\
\hline 8.0 & 0.072617 \\
\hline 9.0 & 0.064778 \\
\hline 10.0 & 0.058455 \\
\hline 11.0 & 0.053249 \\
\hline 12.0 & 0.048889 \\
\hline
\end{tabular}

When the tip speed ratio equals about 0.635428 , there is a peak value of about 0.401017 in the curve, which is the torque limit. As can be seen from Table 2, when the tip speed ratio is greater than 6 , the torque coefficient of the high-speed wind turbine in steady operation state will not exceed 0.1 . This result based on the hypothesis that the wind turbine has the ideal chord length and is running in ideal fluid environment (drag $C_{D}=0$ ), so it is sure that the torque coefficient of the real high-speed wind turbine in steady operation state will be smaller than 0.1 .

Equation (15) may also be derived with the highest performance formula of power in reference [3], because the torque coefficient is equal to the power coefficient divided by the tip speed ratio. The rationality of the highest performance formula of power was proved by the deducing process that the power coefficient approaches Betz limit if its tip speed ratio approaches infinity, and thus the torque coefficient rationality is obvious.

\section{CONCLUSIONS}

(1) For the wind turbine in steady operation state, there is a theoretical limit of the torque coefficient, 0.401 , which is the highest boundary that any actual wind turbine with any tip speed ratio can not be crossed.

(2) For the wind turbine in steady operation state, the highest performance of the torque associated with the tip speed ratio determined by the equation (15), which is lower boundary that any actual wind turbine with same tip speed ratio can not be crossed.

(3) For the high-speed wind turbine which tip speed ratio is greater than 6 , the torque coefficient in steady operation state is unlikely to exceed 0.1 .

(4) As a theory reference value of the actual design, the actual wind turbine torque coefficient can be pre-estimate by the formula (14) or Table 1.

\section{REFERENCES}

[1] Bergey K H. The Lanchester- Betz limit. Journal of Energy, 1979; 3 : 382-384.

[2] van Kuik G A M, The Lanchester- Betz- Joukowsky Limit. Wind Energy. 2007(10): 289-291.

[3] Haibo JIANG, Shuliang CAO, Ping YANG. Power Limit of Horizontal Axis Wind Turbine. Chinese Journal of Mechanical Engineering, 2011, 47 (10): 113-118. (in Chinese)

[4] Tony Burton, David Sharpe, Nick Jenkins, et al. Wind Energy Handbook. John Wiley \& Sons Ltd, 2005.

[5] Martin O L Hansen. Aerodynamics of Wind Turbines (2nd ed). Beijing: China electric power press. 2009. (in Chinese)

[6] HE DEXIN, et al. Wind project and industry aerodynamics. Beijing: National Defense Industry Press, 2006 (in Chinese) 